\title{
Chemically-specific time-resolved surface photovoltage spectroscopy: carrier dynamics at the interface of quantum dots attached to a metal oxide
}

\author{
Ben F. Spencer ${ }^{\text {a,b,*, }}$, Matthew J. Cliffe ${ }^{\text {a,b }}$, Darren M. Graham ${ }^{\text {a }}$, Samantha J. O. Hardman ${ }^{\text {c }}$, Elaine A. \\ Seddon ${ }^{\mathrm{a}, \mathrm{b}}$, Karen L. Syres ${ }^{\mathrm{d}}$, Andrew G. Thomas ${ }^{\mathrm{a}}$, Fausto Sirotti ${ }^{\mathrm{e}}$, Mathieu G. Silly ${ }^{\mathrm{e}}$, Javeed Akhtar ${ }^{\mathrm{f}, \mathrm{g}}$, \\ Paul O’Brien ${ }^{\text {f }}$, Simon M. Fairclough ${ }^{\text {h }}$, Jason M. Smith ${ }^{\text {i }}$, Swapan Chattopadhyay ${ }^{\text {b }}$, Wendy R. Flavell ${ }^{\text {a }}$ \\ ${ }^{a}$ School of Physics and Astronomy and the Photon Science Institute, The University of Manchester, Manchester M13 9PL, United \\ Kingdom. \\ ${ }^{\mathrm{b}}$ The Cockcroft Institute, Sci-Tech Daresbury, Keckwick Lane, Daresbury, Warrington WA4 4AD, Cheshire, United Kingdom. \\ ${ }^{c}$ Manchester Institute of Biotechnology, Faculty of Life Sciences, University of Manchester, 131 Princess Street, Manchester M1 7DN, \\ United Kingdom. \\ ${ }^{\mathrm{d}}$ School of Chemistry, The University of Nottingham, University Park, Nottingham NG7 2RD, United Kingdom. \\ ${ }^{\mathrm{e}}$ Synchrotron SOLEIL, BP 48, Saint-Aubin, F91192 Gif sur Yvette CEDEX, France. \\ ${ }^{\mathrm{f}}$ Department of Chemistry, University of Manchester, Oxford Road, Manchester M13 9PL, United Kingdom. \\ ${ }^{g}$ Department of Physics, Nano-Science \& Materials Synthesis Laboratory, COMSATS Institute of Information Technology, Chakshahzad \\ Park Road, Islamabad 44000, Pakistan. \\ ${ }^{\mathrm{h}}$ Department of Chemistry, University of Oxford, South Parks Road, Oxford OX1 3QR, United Kingdom. \\ ${ }^{\mathrm{i}}$ Department of Materials, University of Oxford, Parks Road, Oxford OX1 3PH, United Kingdom. \\ *E-mail: ben.spencer@manchester.ac.uk
}

Keywords: Surface photovoltage, Time-resolved photoemission, Metal oxides, Semiconductor Surface, Carrier Dynamics, Photovoltaics, Colloidal Quantum Dots

\begin{abstract}
We describe a new experimental pump-probe methodology where a 2D delay-line detector enables fast (ns) monitoring of a narrow XPS spectrum in combination with a continuous pump laser. This has been developed at the TEMPO beamline at Synchrotron SOLEIL to enable the study of systems with intrinsically slow electron dynamics, and to complement faster measurements that use a fs laser as the pump. We demonstrate its use in a time-resolved study of the surface photovoltage of the $m$-plane $\mathrm{ZnO}$ (10 $\overline{10}$ ) surface which shows persistent photoconductivity, requiring monitoring periods on ms timescales and longer. We make measurements from this surface in the presence and absence of chemically-linked quantum dots (QDs), using type I PbS and type II $\mathrm{CdSe} / \mathrm{ZnSe}$ (core/shell) QDs as examples. We monitor signals from both the ZnO substrate and the bound QDs during photoexcitation, yielding evidence for charge injection from the QDs into the ZnO. The chemical specificity of the technique allows us to observe differences in the extent to which the QD systems are influenced by the field of the surface depletion layer at the $\mathrm{ZnO}$ surface, which we attribute to differences in the band structure at the interface.
\end{abstract}

\section{Introduction}

The study of 'real-time' charge carrier dynamics at material interfaces is becoming ever more important as next generation technologies increasingly access nanoscale dimensions. This is particularly the case for solar harvesting technologies, where a clear understanding of the dynamics of photoexcited carriers is of key fundamental importance. One such example is in devices utilising cost-reducing photovoltaic materials such as light-harvesting colloidal quantum dots (QDs) [1, 2] where a transparent conducting oxide (TCO) such as $\mathrm{ZnO}$ is used as photoanode [3-5]. These QDs have been shown to exhibit multiple exciton generation (MEG, creation of more than one pair of charge carriers from a single photon) for photon energies greater than around twice the effective band gap. The band gap is typically $1.0 \mathrm{eV}$, meaning MEG is present at wavelengths within the solar spectrum, giving potential for photovoltaic devices with enhanced efficiencies [1]. Considerable effort is currently being devoted to extending the photoexcited electron-hole pair lifetime in order to maximise the benefit of MEG. Quantum dot cores (known as type I QDs) that have been surrounded by a shell are known as type II QDs, and these are engineered such that the band structure leads to the photoexcited electrons and holes being separately trapped in the core and shell or vice versa, reducing the wavefunction overlap and extending radiative recombination lifetimes. Hence there is great interest in these type II structures for photovoltaic applications [6-8]. Here we study the carrier dynamics in heterojunctions formed both from type II QDs, and from type I QDs that have been shown to exhibit MEG, attached to a semiconductor photoanode, $\mathrm{ZnO}$.

Carrier dynamics at the surface of semiconductors may be probed using time-resolved surface photovoltage (SPV) spectroscopy [9-11]. The space charge layer at the surface of semiconductors where the Fermi level is pinned by surface states within the band gap leads to band bending in this region. For $n$-type semiconductors the bands bend upwards due to the presence of a depletion layer, and downwards in $p$-type semiconductors due to an accumulation layer. A laser is used to promote carriers across the band gap, and, in the 
case of an $n$-type semiconductor, electrons and holes migrate away from and towards the surface respectively (and vice versa in a $p$-type semiconductor). This additional electric field reduces the band bending and changes the energy of the valence band maximum in the space charge region. A synchronised X-ray source is then used to excite core level photoelectrons (using X-ray photoelectron spectroscopy, XPS), as the binding energy (BE) of the core levels in the space charge region at the surface shift as the valence band maximum shifts [9-11]. In $n$ type semiconductors these core levels shift to higher BE and in $p$-type semiconductors to lower BE [10]. By varying the time delay between the laser pump and XPS probe, the recombination dynamics is monitored over time, because the band bending and core level binding energies return to equilibrium as carriers recombine. Thus the recombination dynamics at the surface are elucidated [10-18].

For the case of colloidal QDs chemically linked to a semiconductor, this technique then provides a way to monitor the carrier dynamics in the semiconductor substrate upon photoexcitation of the QDs, i.e., as additional carriers are injected into the semiconductor conduction band upon laser illumination of the QDs [19]. Moreover, photoemission allows chemical specificity because the substrate and QDs can be monitored separately or in some cases simultaneously, simply by monitoring different core level photoemission lines. The technique then gives a chemically-specific diagnostic of charge transfer between the QD and the substrate.

Laser-pump X-ray-probe SPV experiments using pulsed lasers, where the pump laser is synchronized to individual X-ray pulses from a synchrotron, are limited by the time window available, determined by the synchrotron pulse length and repetition rate [11-13]. In the case of work by us at the SRS, UK, with the synchrotron operating in single-bunch mode, the maximum time window available was $320 \mathrm{ns,} \mathrm{i.e.,} \mathrm{laser} \mathrm{pump}$ and X-ray probe pulses were incident every $320 \mathrm{~ns}$ with a set delay time between the two [10]. When measuring the transient SPV at the Si (111) 7x7 surface the dark carrier lifetime was in excess of the time window, and so a multi-pulse model was required to fit the carrier dynamics since the observed SPV transient was influenced by the residual SPV induced by preceding laser pump pulses [10]. Recently the use of angle-resolved time-of-flight (ARToF) analysers (such as at the BL07LSU beamline at SPring-8, Japan [14]), has allowed synchronisation of the pump laser pulse with hybrid mode pulses in multiple synchrotron periods, enabling SPV measurements extending to $\mu$ s timescales, in studies of $\mathrm{Si}$ (111) [14-16], $\mathrm{ZnO}$ (0001) [17], and $\mathrm{TiO}_{2}$ (110) [18].

A different approach is necessary to enable SPV measurements over much longer timescales (up to ms), which may be required where the electron dynamics are intrinsically slow, for example due to persistent photoconductivity (PPC) as in $\mathrm{ZnO}$. This has been enabled at the TEMPO beamline at Synchrotron SOLEIL, France [20], where pump-probe measurements are now possible over timescales ranging from $50 \mathrm{ps}$ to ms or longer. Fast (ps - ns) measurements use a pulsed fs laser in combination with hybrid mode synchrotron radiation [21]. Dynamics with slower characteristic times are conveniently monitored using a CW laser. This is modulated using the output of a signal generator, where the period and duty cycle are easily controlled, and a fast XPS detection system is used to monitor XPS spectra at small time intervals over this modulation period [21], e.g. a core-level photoemission peak is monitored at $50 \mathrm{~ns}$ intervals as the CW laser is switched on and then off over ms timescales (typically on for $0.5 \mathrm{~ms}$, and off for $0.5 \mathrm{~ms}$ ). The key advantage of this system is the access this affords to long monitoring periods, from $\mu \mathrm{s}$ to $\mathrm{ms}, \mathrm{s}$ and beyond. This is important for the SPV at the

$\mathrm{ZnO}$ (10 $\overline{10}$ ) surface where ms dynamics are exhibited, due to PPC controlled by trapping at band gap states associated with ionized oxygen vacancies $[10,19,22,23]$.

\section{Experiment}

In order to access relatively wide time domains in pump-probe XPS spectroscopy experiments, a twodimensional (2D) delay-line detector was installed on a SCIENTA SES 2002 analyser at the TEMPO beamline at SOLEIL $[21,24]$. This allows XPS spectra over a narrow BE range $(\sim 2 \mathrm{eV})$ to be captured at least every 50 ns. The delay-line detector was developed in combination with custom-built software in order to monitor XPS spectra over time windows easily controlled by a signal generator. The triggering of this fast XPS detector is also used to control and modulate a CW laser (Coherent CUBE, $10 \mathrm{~mW}, 372 \mathrm{~nm}$ ). The complete experimental setup is shown in Fig. 1. The laser is modulated as fast XPS spectra are captured every 50 ns over the period, $T$, of the laser modulation, e.g., for a period of $1 \mathrm{~ms}$ with a 50\% duty cycle, 20,000 XPS spectra are captured as the laser is on for $0.5 \mathrm{~ms}$ and off for $0.5 \mathrm{~ms}$. When the laser is switched on, the SPV shift is monitored using a shifting core level photoemission peak as charge carriers are injected into the conduction band, and a new equilibrium BE is established. The recombination time of these charge carriers is measured when the laser is turned off. Whilst the time resolution of the delay-line detector is $5 \mathrm{~ns}$ [21], the time resolution of the detection system is limited by the time spread of photoelectrons within the analyser and the speed of the electronics, which has been determined to be $150 \mathrm{~ns}$. The signal-to-noise ratio (SNR) of a single XPS spectrum is low, and so typically in excess of 10,000 accumulations are taken (over 2-3 hours of data collection time) to obtain an adequate SNR. Very small SPV shifts on the order of meV changes in binding energy are easily detected under these conditions as shown in Fig. 2. 
X-ray photon energies of $200 \mathrm{eV}$ (for the $\mathrm{Zn} 3 d$ core level) and $280 \mathrm{eV}$ (for the $\mathrm{Pb} 4 f$ core level) were chosen to maximize surface sensitivity (for examination of the space charge layer) without undue loss of flux. High-quality XPS spectra of the monitored photoemission peaks were taken in static mode in order to extract peak fitting parameters (e.g., spin-orbit splitting, full width at half maximum (FWHM)). These parameters were then applied to each fast XPS spectrum across the laser modulation time window, and the BE positions of the photoemission peaks obtained from the fitting were used to measure the SPV shift upon, during and after photoexcitation. Static XPS with and without laser illumination was also used to ensure that no long-term sample charging was occurring, i.e., that the photoemission peaks returned to the original BE positions after the laser was turned off.

Spatial overlap of the laser pump and X-ray probe beam at the sample position was achieved using a charge-coupled device (CCD) camera, and a photodiode was scanned across the sample position to measure the sizes of the laser pump and X-ray probe beams. The X-ray beam measured approximately $150 \mu \mathrm{m}$ vertically by $100 \mu \mathrm{m}$ horizontally, and the laser beam radius was approximately $3 \mathrm{~mm}$. Ensuring the photoexcitation beam is substantially larger than the X-ray probe ensures an even photoexcitation fluence across the area being probed.

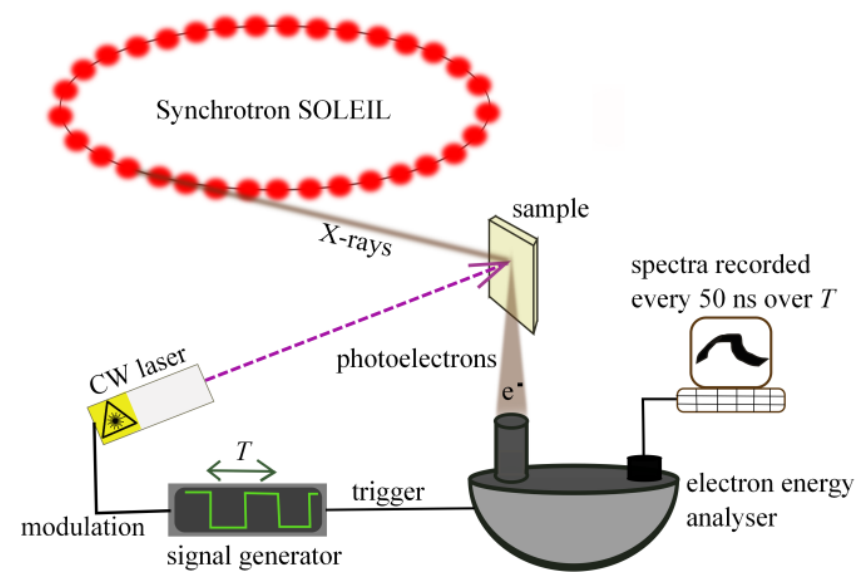

Fig. 1. Schematic diagram of the laser-pump X-ray-probe experiment at the TEMPO beamline at Synchrotron SOLEIL [20, 21]. A signal generator is used to modulate a continuous wave (CW) laser over a period $T$ (Coherent CUBE, $10 \mathrm{~mW}, 372 \mathrm{~nm}$ ), and fast XPS spectra are captured every $50 \mathrm{~ns}$ over the modulation period using a 2D delay-line detector installed on a SCIENTA SES 2002 electron energy analyser [21]. The signal generator is used both to trigger the detection period and to control the time interval between spectra. Typically in excess of 10,000 accumulations over the modulation period are collected to obtain an adequate signal-to-noise ratio.

\section{Materials}

The non-polar $m$-plane $\mathrm{ZnO}$ (10 $\overline{10}$ ) surface was prepared according to an established recipe as has been detailed elsewhere $[10,19]$. The final part of the cleaning procedure includes a period of annealing in $10^{-7} \mathrm{mbar}$ $\mathrm{O}_{2}$ at $700 \mathrm{~K}$, which is required to heal an excess of oxygen vacancies created by previous argon ion sputtering and electron bombardment annealing cycles. This oxygen annealing period controls the concentration of oxygen vacancies, which is critical in controlling the final conductivity of the surface and the timescales for PPC. A change in the length of the oxygen anneal (for example from ten to twenty minutes) changes the dark carrier lifetime substantially $[10,19]$. The SPV at the $\mathrm{ZnO}$ surface was monitored using the $\mathrm{Zn} 3 d$ core level.

Two colloidal QD samples, type I PbS [2] and type II CdS/ZnSe core/shell QDs [19], were chemicallylinked to a clean $\mathrm{ZnO}$ substrate using 3-MPA (3-mercaptopropionic acid) ligands, where the thiol links to the QD ( $\mathrm{Pb}$ atoms in $\mathrm{PbS} \mathrm{QDs}, \mathrm{Zn}$ atoms in the $\mathrm{ZnSe}$ shell in CdS/ZnSe QDs) and the acid group to $\mathrm{Zn}$ in the substrate. In the $\mathrm{PbS} \mathrm{QD}$ experiment, the substrate was initially prepared with a twenty minute oxygen anneal cycle, and for the CdS/ZnSe QD experiment the substrate was first prepared with a ten minute oxygen anneal. The preparation and characterisation of the QDs have been detailed elsewhere [19]. The type I (core-only) PbS QD sample used has been shown to exhibit MEG for photon energies greater than $\sim 2.5$ times the effective band gap [1]. The PbS QD sample was monitored using XPS of the Pb $4 f$ core level, whilst the CdS/ZnSe QD was monitored simultaneously with the $\mathrm{ZnO}$ substrate due to the presence of zinc in both substrate and QD; a $\mathrm{Zn} 3 d$ species associated with the QD (chemically shifted to a higher BE) was fitted along with the dominant $\mathrm{Zn} 3 d$ peak associated with the substrate.

The laser photon energy $(3.33 \mathrm{eV})$ was chosen for photoexcitation of the $\mathrm{ZnO}$ substrate and for photoexcitation of the $\mathrm{PbS}$ QDs and the ZnSe shell of the CdS/ZnSe QDs. For the PbS sample, this photon energy means that MEG is present since the photon energy is greater than 3 times the effective band gap.

\section{Results}


The SPV at the $\mathrm{ZnO}(10 \overline{10})$ surface upon laser photoexcitation was monitored with and without chemically-linked $\mathrm{PbS}$ and $\mathrm{CdS} / \mathrm{ZnSe} \mathrm{QDs}$. The energy level line up diagrams for these two QD samples at the surface of $\mathrm{ZnO}$ are shown in Figs. 2 (a) and (e). The transient SPV, $\Delta \mathrm{V}_{\mathrm{SP}}(t)$, is fitted with a decelerating exponential decay as detailed elsewhere $[10,12,13,19]$ :

$$
\Delta \mathrm{V}_{\mathrm{SP}}(t)=-\alpha k T \ln \left[1-\exp \left(\frac{-t}{\tau_{\infty}}\right)\left(1-\exp \left(\frac{-\Delta \mathrm{V}_{\mathrm{SP}}^{\mathrm{tot}}}{\alpha k T}\right)\right)\right]
$$

Here, $\alpha$ is a material parameter likened to the ideality factor in a Schottky diode [9], $\tau_{\infty}$ is the dark carrier lifetime (the lifetime of carriers in the absence of a SPV), and $\Delta \mathrm{V}_{\mathrm{SP}}^{\text {tot }}$ is the total SPV shift.
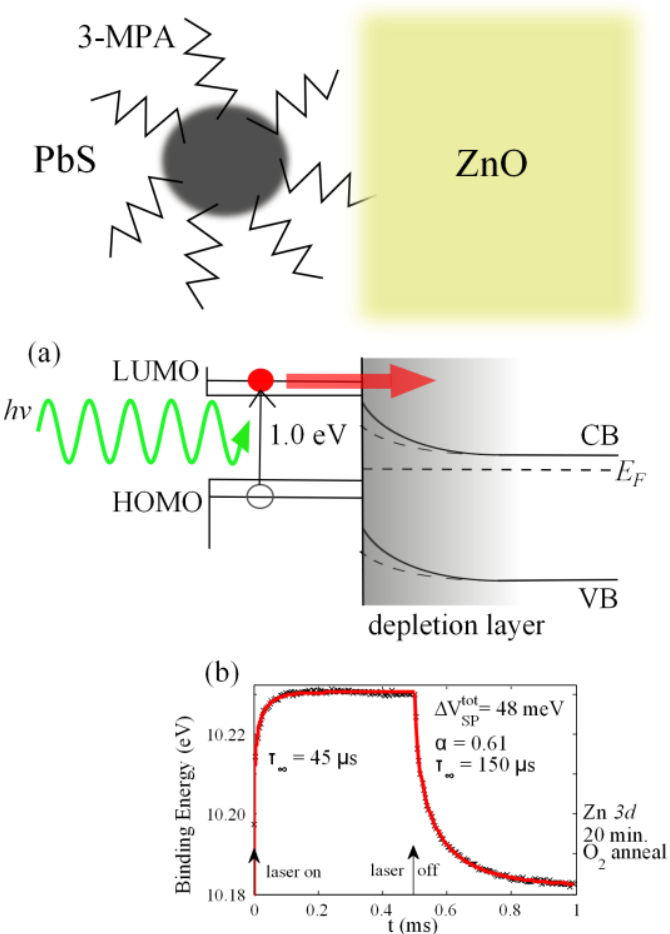

(c) $\mathrm{Zn} 3 d$ - substrate

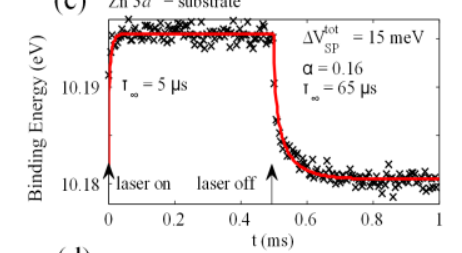

(d) $\mathrm{Pb} 4 f-\mathrm{QD}$

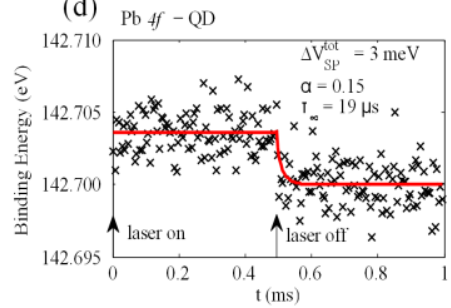

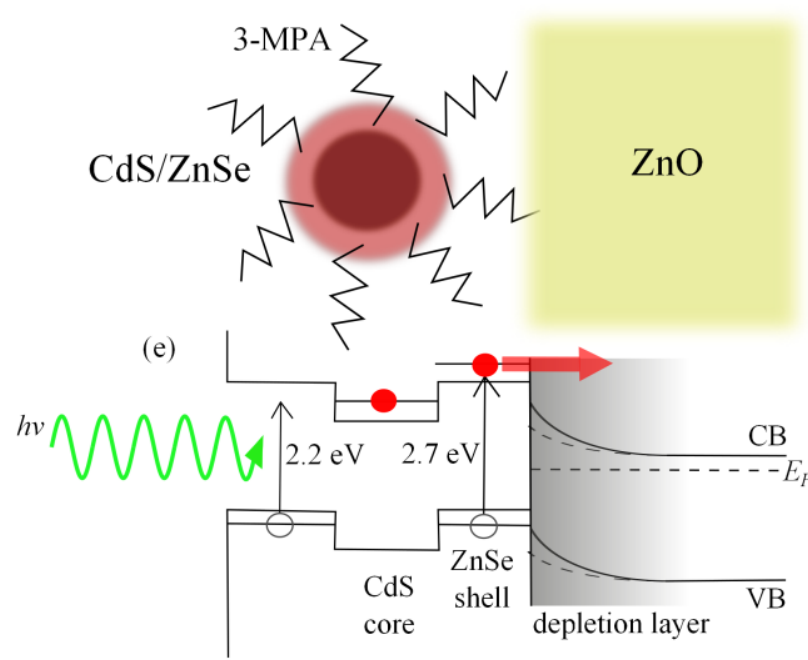

Clean $\mathrm{ZnO}$

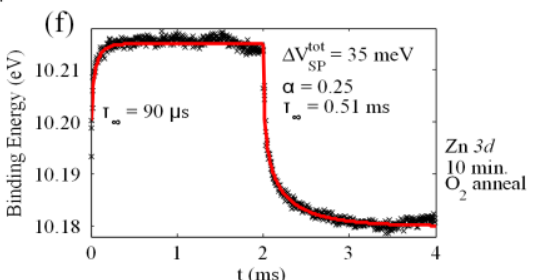

(g) Zn $3 d$ - substrate component

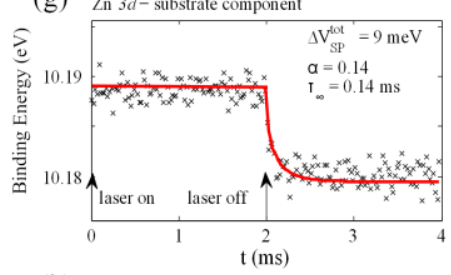

(h) $\mathrm{Zn} 3 d$ - ligand $\mathrm{QD}$ component

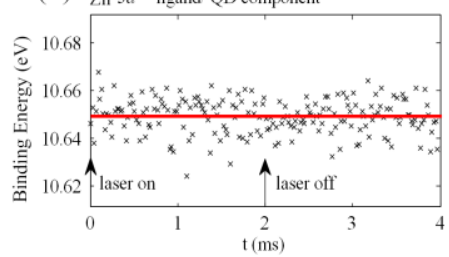

Fig. 2. Transient surface photovoltage (SPV) of quantum dots (QDs) chemically linked to $\mathrm{ZnO}$ ( $10 \overline{10}$ ) with 3-MPA (3-mercaptopropionic acid) ligands. The parameters extracted from fitting are shown. An X-ray photon energy of $200 \mathrm{eV}$ was used for the $\mathrm{Zn} 3 d$ core level (b, c, f$\mathrm{h}$ ), and a photon energy of $280 \mathrm{eV}$ was used for the $\mathrm{Pb} 4 f$ core level $(\mathrm{d})$. Arrows denote laser modulation; the laser is turned on at $\mathrm{t}=0 \mathrm{~ms}$, and switched off at $T / 2$, where the period $T$ is $1 \mathrm{~ms}$ for the PbS QD case, and $4 \mathrm{~ms}$ for the CdS/ZnSe QD case. In all cases laser photoexcitation at $372 \mathrm{~nm}(h v=3.33 \mathrm{eV})$ was used, meaning that both QDs and the $\mathrm{ZnO}$ substrate were photoexcited (and for the CdS/ZnSe QDs, the shell of the QDs was photoexcited directly, enabling charge injection from the shell into the substrate as illustrated in (e)). Energy level diagrams (a) and (e) are adapted from [25, 26]. Dashed lines indicate a reduction in the band bending upon photoexcitation as carriers are promoted (or injected) into the $\mathrm{CB}$. 
Type I (core only) PbS QDs: (a) energy level line up diagram of the highest occupied molecular orbital (HOMO) and lowest unoccupied molecular orbital (LUMO) of the QDs with the $\mathrm{ZnO}$ conduction band (CB), valence band (VB) and Fermi level ( $\left.E_{F}\right)$; (b) transient SPV for the clean $\mathrm{ZnO}$ substrate, prepared with a twenty minute oxygen anneal cycle; (c) transient SPV of the Zn $3 d$ core level after QD attachment; (d) transient $\mathrm{SPV}$ of the $\mathrm{Pb} 4 f$ core level of the $\mathrm{PbS}$ QDs.

Type II (core/shell) CdS/ZnSe QDs: (e) energy level line up diagram, showing that for photon energies less than $2.7 \mathrm{eV}$, electrons and holes are trapped in the core and shell of the QD respectively; (f) transient SPV for the clean substrate. A shorter oxygen anneal cycle of ten minutes was used, giving an extended dark carrier lifetime compared with (b); (g) transient SPV of the Zn $3 d$ core level component associated with the substrate (at a binding energy of 10.18 eV) after QDs have been attached to the surface; (h) transient SPV of the Zn $3 d$ core level component associated with the ligand/QD (chemically shifted to a higher binding energy of $\sim 10.65 \mathrm{eV}$ ), where no SPV shift is observed.

Figs. 2 (b) and (f) show that the dark carrier lifetime, $\tau_{\infty}$, extracted from fitting equation (1) increases from $150 \mu \mathrm{s}$ to $510 \mu \mathrm{s}$, and the magnitude of the SPV shift, $\Delta \mathrm{V}_{\mathrm{SPV}}^{\text {tot }}$, decreases from 48 to $35 \mathrm{meV}$, as the oxygen anneal cycle length is halved, i.e., the dynamics are slowed as the oxygen vacancy concentration in the substrate is increased (as oxygen annealing acts to reduce the number of oxygen vacancies in the naturally nonstoichiometric $\mathrm{ZnO}_{1-x}$ [10]). The timescale of the onset of the SPV upon laser illumination, modelled in an analogous way to the SPV decay (Eq. (1)), also increases from 45 to $90 \mu \mathrm{s}$.

Figs. 2 (c) and (g) show the changes in the carrier dynamics at the $\mathrm{ZnO}$ surface when the QD samples have been chemically linked to the surface. In both cases, the dynamics at the substrate surface speed up, even though the magnitude of the total SPV shift is reduced. For the PbS case, the total SPV shift is reduced to $15 \mathrm{meV}$, the dark carrier lifetime is reduced to $65 \mu \mathrm{s}$, and the SPV onset time is reduced to $5 \mu \mathrm{s}$. For the CdS/ZnSe case, the total SPV shift is reduced to $9 \mathrm{meV}$, the dark carrier lifetime is reduced to $140 \mu \mathrm{s}$, and the onset time appears instantaneous, i.e., the measured onset time is now limited by the time resolution of the experiment $(0.15 \mu \mathrm{s})$.

Figs. 2 (d) and (h) show the transient SPV shifts now recorded using a core level photoemission line associated with the QDs rather than the substrate: in (d) the Pb $4 f$ core level for the PbS QDs, and in (h) a Zn $3 d$ core level species chemically-shifted to higher binding energy, associated with $\mathrm{Zn}$ atoms in the QDs attached to the ligand [19]. The $\mathrm{Zn} 3 d$ core level associated with the CdS/ZnSe QDs does not shift upon photoexcitation, whereas a small but clear SPV shift of $3 \mathrm{meV}$ is observed in the PbS QDs, with a dark carrier lifetime of $19 \mu \mathrm{s}$. The onset time of this SPV shift is also within the time resolution of the experiment, appearing as an instantaneous shift at $\mathrm{t}=0 \mathrm{~ms}$.

\section{Discussion}

The change in the magnitude and carrier recombination lifetimes for the clean $\mathrm{ZnO}$ substrate with two different oxygen anneal cycle lengths (20 minutes in Fig. 2 (b) and 10 minutes in Fig. 2 (f)) is consistent with the PPC being controlled by the oxygen vacancy concentration; a longer oxygen anneal cycle reduces the oxygen vacancy concentration. Dark carrier lifetimes recorded under similar conditions, but in different experiments are found to be reproducible to around a factor of two [10]. Band gap states associated with ionized oxygen vacancies have been implicated in this PPC, with recent experimental evidence supporting the theory proposed by Lany and Zunger [10, 19, 22, 23]; in this scenario, the PPC persists for longer as the number of oxygen vacancies (which, in their unionized state, act as efficient hole traps) increases [10].

Deposition of QDs chemically linked to the surface with 3-MPA ligands reduces the total SPV shifts observed in the $\mathrm{ZnO}$ substrate, but also reduces the dark carrier lifetimes and the onset times of the SPV shifts (Figs. 2 (c) and (g)). The presence of QDs linked to the surface is expected to change the equilibrium band bending, $V_{0}$, if the QDs donate or withdraw charge from the surface, and the total SPV shift, $\Delta \mathrm{V}_{\mathrm{SP}}^{\text {tot }}$, depends on the equilibrium band bending according to [27]:

$$
\frac{\Delta \mathrm{V}_{\mathrm{SP}}^{\mathrm{tot}}}{k T} \exp \left(\frac{\Delta \mathrm{V}_{\mathrm{SP}}^{\mathrm{tot}}}{k T}\right)=\frac{n_{P}}{n_{0}} \exp \left(\frac{V_{0}}{k T}\right),
$$

where $n_{P}$ and $n_{0}$ are the photoexcited carrier density and the doping carrier concentration respectively. In all cases the laser photoexcitation fluence was the same, indicating a change in equilibrium band bending. The reduction in the total SPV shift observed on QD deposition in both cases here suggests that the QDs donate electrons into the $\mathrm{ZnO}$ surface. In the absence of any new mechanism for carrier injection or recombination, this reduction in the total SPV shift is expected to give rise to an increase in carrier lifetime, rather than the decrease observed in Figs. 2 (c) and (g), since (for carriers recombining by thermionic emission across the surface depletion layer) the photoexcited carrier lifetime, $\tau$, varies with SPV shift, $\Delta \mathrm{V}_{\mathrm{SP}}$, according to [12]: 


$$
\tau=\tau_{\infty} \exp \left(\frac{-\Delta \mathrm{V}_{\mathrm{SP}}}{\alpha \mathrm{kT}}\right) .
$$

In this model, the dynamic change in the photoexcited carrier lifetime with SPV shift requires the decelerating exponential model given in Eq. (1), where the lifetime increases as SPV shift is reduced. The fact that the onset times for the SPV shifts are faster, rather than slower, when the equilibrium band bending is reduced by attachment of QDs suggests direct charge injection from the QDs to the ZnO conduction band (CB) as anticipated according to the energy level line up diagrams shown in Figs. 2 (a) and (e). In the case of CdS/ZnSe QDs the onset appears instantaneous suggesting that the onset time is now within the experimental time resolution, and indeed there is evidence that charge transfer to an oxide substrate in similar QD systems may occur on ps and fs timescales [28-30]. The reduced dark carrier lifetime may indicate that the QD attachment is providing additional pathways for electron-hole pair recombination that avoids the long-lived PPC state (e.g. via new interface states). These changes in the recombination dynamics are important when considering light harvesting QDs with $\mathrm{ZnO}$ as photoanode as model systems for next-generation photovoltaics.

Transient SPV shifts upon laser illumination are caused by a change in the band bending at the interface, which is caused by the presence a depletion layer in the case of $n$-type $\mathrm{ZnO}$. In the case of QDs, which are nmscale semiconductor crystals, intrinsic band bending is not necessarily expected as the size of the QDs is much less than typical depletion layer widths. Therefore, no intrinsic SPV shift is expected when monitoring core level photoemission lines associated with the QDs. However, the electric field associated with the surface depletion layer in the $\mathrm{ZnO}$ substrate permeates the medium both above and below the surface, to an extent dependent on factors such as the dielectric constant and carrier concentration of the medium. We might expect to observe a SPV shift in the core levels of atoms associated with the QD if those atoms lie within this field; the size would depend on the position of the atom in the field, but is clearly expected to be smaller than the shift we observe at the $\mathrm{ZnO}$ surface. It is interesting that a small SPV shift of $3 \mathrm{meV}$ (in the same direction as the $\mathrm{ZnO}$ substrate, i.e. to higher $\mathrm{BE}$ ) is observed when monitoring the $\mathrm{Pb} 4 f$ photoemission line in the $\mathrm{PbS}$ QD sample (Fig. 2 (d)). This indicates that the $\mathrm{Pb}$ atoms in the QD lie in the tail of the field associated with the surface depletion layer in the $\mathrm{ZnO}$ substrate. Analysis of this transient gives an onset time within the time resolution of the experiment, and the decay of the SPV shift yields a fitted dark carrier lifetime of $19 \mu \mathrm{s}$, faster than that observed in the substrate $(65 \mu \mathrm{s})$. Both are consistent with fast intrinsic charge dynamics in $\mathrm{PbS}$, not subject to the PPC observed in ZnO. Interestingly, a recent study by Neppl et al. [31] observed SPV shifts in N3 dye molecules attached to nanoporous $\mathrm{ZnO}$. It is then interesting that we observe no SPV shift in the shell of the $\mathrm{CdS} / \mathrm{ZnSe}$ type II QDs, monitored using a chemically-shifted component of the Zn $3 d$ core level (Fig. 2 (h)); this may be associated with the potential barrier provided by the ZnSe shell, which may act to truncate the field associated with the $\mathrm{ZnO}$ substrate. We note that in this case photoexcited electrons are more stable in either the $\mathrm{ZnO}$ substrate or the $\mathrm{CdS}$ core of the QD than in the ZnSe shell (Fig. 2(e)). The difference in behaviour between the two QD systems points to the need for more experiments in this field, particularly using photon frequencies resonant with the lowest absorption energies of the QDs. However, the measurements presented here show the power of soft X-ray pump-probe experiments in allowing us to probe electron dynamics at these complex interfaces with atomic specificity.

\section{Conclusions}

A fast XPS technique, where a 2D delay-line detector enables fast (ns) monitoring of a narrow XPS spectrum at the TEMPO beamline at Synchrotron SOLEIL [20, 21], in combination with a CW laser, has been developed to enable the study of systems with intrinsically slow electron dynamics. This has allowed the charge carrier dynamics at the interface of colloidal QDs chemically-linked to $\mathrm{ZnO}(10 \overline{10})$ to be monitored on ms timescales, required due to the PPC at the $\mathrm{ZnO}(10 \overline{10})$ surface $[10,19,22,23]$. The shift in core level photoemission lines upon photoexcitation, the SPV shift, has been measured over time windows of several ms. The chemical specificity of XPS allows for monitoring of not only the substrate but also of the QDs bound to the surface. In the case of PbS QDs, the observations of a small SPV shift of the $\mathrm{Pb} 4 f$ core level indicates that the QDs lie within the field associated with the depletion layer at the $\mathrm{ZnO}$ surface. However, no transient SPV is observed when monitoring a Zn $3 d$ core level component associated with the shell of type II CdS/ZnSe QDs, which may be due to the effect of the potential barrier at the QD surface provided by this shell. While further study is required to better understand the charge transfer and recombination dynamics at these interfaces, our work highlights the power of laser-pump X-ray probe techniques in measuring photoexcited carrier dynamics at these interfaces with chemical specificity.

\section{Acknowledgments}


The research leading to these results received funding from the European Community's Seventh Framework Programme (FP7/2007-2013) under grant agreement $n^{\circ}$ 226716, allowing access to Synchrotron SOLEIL. Work was also supported by EPSRC (UK) under grant $\mathrm{n}^{\circ} \mathrm{EP} / \mathrm{K} 008544 / 1$ and the Cockcroft Institute via its STFC core grant ST/G008248/1.

\section{References}

[1] S.J.O. Hardman, D.M. Graham, S.K. Stubbs, B.F. Spencer, E.A. Seddon, H.T. Fung, S. Gardonio, F. Sirotti, M.G. Silly, J. Akhtar, P. O'Brien, D.J. Binks, W.R. Flavell, Electronic and surface properties of PbS nanoparticles exhibiting efficient multiple exciton generation, Physical Chemistry Chemical Physics, 13 (2011) 20275.

[2] J. Akhtar, M.A. Malik, P. O'Brien, K.G.U. Wijayantha, R. Dharmadasa, S.J.O. Hardman, D.M. Graham, B.F. Spencer, S.K. Stubbs, W.R. Flavell, D.J. Binks, F. Sirotti, M. El Kazzi, M. Silly, A greener route to photoelectrochemically active PbS nanoparticles, Journal of Materials Chemistry, 20 (2010) 2336.

[3] P.D.C. King, T.D. Veal, Conductivity in transparent oxide semiconductors, Journal of Physics-Condensed Matter, 23 (2011) 334214.

[4] C. Klingshirn, J. Fallert, H. Zhou, J. Sartor, C. Thiele, F. Maier-Flaig, D. Schneider, H. Kalt, 65 years of ZnO research - old and very recent results, Physica Status Solidi B-Basic Solid State Physics, 247 (2010) 1424.

[5] B. Carlson, K. Leschkies, E.S. Aydil, X.Y. Zhu, Valence band alignment at cadmium selenide quantum dot and zinc oxide (10(1)over-bar0) interfaces, Journal of Physical Chemistry C, 112 (2008) 8419.

[6] V.I. Klimov, S.A. Ivanov, J. Nanda, M. Achermann, I. Bezel, J.A. McGuire, A. Piryatinski, Single-exciton optical gain in semiconductor nanocrystals, Nature, 447 (2007) 441.

[7] A. Piryatinski, S.A. Ivanov, S. Tretiak, V.I. Klimov, Effect of quantum and dielectric confinement on the exciton-exciton interaction energy in type II core/shell semiconductor nanocrystals, Nano Letters, 7 (2007) 108.

[8] S. Kim, B. Fisher, H.J. Eisler, M. Bawendi, Type-II quantum dots: CdTe/CdSe(core/shell) and $\mathrm{CdSe} / \mathrm{ZinTe}$ (core/shell) heterostructures, Journal of the American Chemical Society, 125 (2003) 11466.

[9] L. Kronik, Y. Shapira, Surface photovoltage phenomena: theory, experiment, and applications, Surface Science Reports, 37 (1999) 1.

[10] B.F. Spencer, D.M. Graham, S.J.O. Hardman, E.A. Seddon, M.J. Cliffe, K.L. Syres, A.G. Thomas, S.K. Stubbs, F. Sirotti, M.G. Silly, P.F. Kirkham, A.R. Kumarasinghe, G.J. Hirst, A.J. Moss, S.F. Hill, D.A. Shaw, S. Chattopadhyay, W.R. Flavell, Time-resolved surface photovoltage measurements at n-type photovoltaic surfaces: $\mathrm{Si}(111)$ and $\mathrm{ZnO(10(1)over-bar0),} \mathrm{Phys} \mathrm{Rev} \mathrm{B,} 88$ (2013) 195301.

[11] J.P. Long, H.R. Sadeghi, J.C. Rife, M.N. Kabler, Surface Space-Charge Dynamics and Surface Recombination on Silicon(111) Surfaces Measured with Combined Laser and Synchrotron Radiation, Phys Rev Lett, 64 (1990) 1158.

[12] W. Widdra, D. Bröcker, T. Gießel, I.V. Hertel, W. Kruger, A. Liero, F. Noack, V. Petrov, D. Pop, P.M. Schmidt, R. Weber, I. Will, B. Winter, Time-resolved core level photoemission: surface photovoltage dynamics of the $\mathrm{SiO} 2 / \mathrm{Si}(100)$ interface, Surf Sci, 543 (2003) 87.

[13] D. Bröcker, T. Gießel, W. Widdra, Charge carrier dynamics at the SiO2/Si(100) surface: a time-resolved photoemission study with combined laser and synchrotron radiation, Chemical Physics, 299 (2004) 247.

[14] M. Ogawa, S. Yamamoto, Y. Kousa, F. Nakamura, R. Yukawa, A. Fukushima, A. Harasawa, H. Kondoh, Y. Tanaka, A. Kakizaki, I. Matsuda, Development of soft X-ray time-resolved photoemission spectroscopy system with a two-dimensional angle-resolved time-of-flight analyzer at SPring-8 BL07LSU, Rev Sci Instrum, 83 (2012)

[15] M. Ogawa, S. Yamamoto, R. Yukawa, R. Hobara, C.H. Lin, R.Y. Liu, S.J. Tang, I. Matsuda, Oscillatory relaxation of surface photovoltage on a silicon surface, Phys Rev B, 87 (2013).

[16] M. Ogawa, R.Y. Liu, C.H. Lin, S. Yamamoto, R. Yukawa, R. Hobara, S.J. Tang, I. Matsuda, Non-linear kinetic model for oscillatory relaxation of the photovoltage effect on a Si(111)7 x 7 surface, Surf Sci, 624 (2014) 70-75.

[17] R. Yukawa, S. Yamamoto, K. Ozawa, M. Emori, M. Ogawa, S. Yamamoto, K. Fujikawa, R. Hobara, S. Kitagawa, H. Daimon, H. Sakama, I. Matsuda, Electron-hole recombination on $\mathrm{ZnO}(0001)$ single-crystal surface studied by time-resolved soft X-ray photoelectron spectroscopy, Appl Phys Lett, 105 (2014).

[18] K. Ozawa, M. Emori, S. Yamamoto, R. Yukawa, S. Yamamoto, R. Hobara, K. Fujikawa, H. Sakama, I. Matsuda, Electron-Hole Recombination Time at TiO2 Single-Crystal Surfaces: Influence of Surface Band Bending, J Phys Chem Lett, 5 (2014) 1953-1957.

[19] B.F. Spencer, M.J. Cliffe, D.M. Graham, S.J.O. Hardman, E.A. Seddon, K.L. Syres, A.G. Thomas, F. Sirotti, M.G. Silly, J. Akhtar, P. O'Brien, S.M. Fairclough, J.M. Smith, S. Chattopadhyay, W.R. Flavell, Dynamics in next-generation solar cells: time-resolved surface photovoltage measurements of quantum dots chemically linked to ZnO (10[1 with combining macron]0), Faraday Discuss, 171 (2014) 275-298. 
[20] F. Polack, M. Silly, C. Chauvet, B. Lagarde, N. Bergeard, M. Izquierdo, O. Chubar, D. Krizmancic, M. Ribbens, J.P. Duval, C. Basset, S. Kubsky, F. Sirotti, TEMPO: a New Insertion Device Beam line at SOLEIL for Time Resolved Photoelectron Spectroscopy Experiments on Solids and Interfaces, AIP proceedings: The 10th International Conference on Synchrotron Radiation Instrumentation, 1234 (2010) 185.

[21] N. Bergeard, M.G. Silly, D. Krizmancic, C. Chauvet, M. Guzzo, J.P. Ricaud, M. Izquierdo, L. Stebel, P. Pittana, R. Sergo, G. Cautero, G. Dufour, F. Rochet, F. Sirotti, Time-resolved photoelectron spectroscopy using synchrotron radiation time structure, J Synchrotron Radiat, 18 (2011) 245-250.

[22] S. Lany, A. Zunger, Anion vacancies as a source of persistent photoconductivity in II-VI and chalcopyrite semiconductors, Phys Rev B, 72 (2005) 035215.

[23] S. Lany, A. Zunger, Dopability, intrinsic conductivity, and nonstoichiometry of transparent conducting oxides, Phys Rev Lett, 98 (2007) 045501.

[24] G. Cautero, R. Sergo, L. Stebel, P. Lacovig, P. Pittana, M. Predonzani, S. Carrato, A two-dimensional detector for pump-and-probe and time resolved experiments, Nuclear Instruments \& Methods in Physics Research Section a-Accelerators Spectrometers Detectors and Associated Equipment, 595 (2008) 447.

[25] S.H. Wei, A. Zunger, Calculated natural band offsets of all II-VI and Ill-V semiconductors: Chemical trends and the role of cation d orbitals, Appl Phys Lett, 72 (1998) 2011-2013.

[26] B.R. Hyun, Y.W. Zhong, A.C. Bartnik, L.F. Sun, H.D. Abruna, F.W. Wise, J.D. Goodreau, J.R. Matthews, T.M. Leslie, N.F. Borrelli, Electron Injection from Colloidal PbS Quantum Dots into Titanium Dioxide Nanoparticles, Acs Nano, 2 (2008) 2206-2212.

[27] J.P. Long, V.M. Bermudez, Band bending and photoemission-induced surface photovoltages on clean nand p-GaN (0001) surfaces, Phys Rev B, 66 (2002) 121308.

[28] W.A. Tisdale, K.J. Williams, B.A. Timp, D.J. Norris, E.S. Aydil, X.Y. Zhu, Hot-Electron Transfer from Semiconductor Nanocrystals, Science, 328 (2010) 1543.

[29] Y. Yang, W. Rodriguez-Cordoba, X. Xiang, T.Q. Lian, Strong Electronic Coupling and Ultrafast Electron Transfer between PbS Quantum Dots and TiO2 Nanocrystalline Films, Nano Letters, 12 (2012) 303.

[30] K. Zidek, K.B. Zheng, C.S. Ponseca, M.E. Messing, L.R. Wallenberg, P. Chabera, M. Abdellah, V. Sundstrom, T. Pullerits, Electron Transfer in Quantum-Dot-Sensitized ZnO Nanowires: Ultrafast TimeResolved Absorption and Terahertz Study, Journal of the American Chemical Society, 134 (2012) 12110.

[31] S. Neppl, A. Shavorskiy, I. Zegkinoglou, M. Fraund, D.S. Slaughter, T. Troy, M.P. Ziemkiewicz, M. Ahmed, S. Gul, B. Rude, J.Z. Zhang, A.S. Tremsin, P.-A. Glans, Y.-S. Liu, C.H. Wu, J. Guo, M. Salmeron, H. Bluhm, O. Gessner, Capturing interfacial photoelectrochemical dynamics with picosecond time-resolved X-ray photoelectron spectroscopy, Faraday Discuss, 171 (2014) 219-241. 